\title{
Entrelacs
}

Cinéma et audiovisuel

11 | 2014

La Voix

\section{Le chant du monde}

Les voix méditées-mélodiées du biographe selon Jean Grémillon

Philippe Roger

\section{OpenEdition}

Journals

\section{Édition électronique}

URL : http://journals.openedition.org/entrelacs/1383

DOI : 10.4000/entrelacs.1383

ISSN : 2261-5482

Éditeur

Éditions Téraèdre

Référence électronique

Philippe Roger, « Le chant du monde », Entrelacs [En ligne], 11 | 2014, mis en ligne le 07 janvier 2015, consulté le 03 mai 2019. URL : http://journals.openedition.org/entrelacs/1383 ; DOI : 10.4000/

entrelacs. 1383

Ce document a été généré automatiquement le 3 mai 2019.

Tous droits réservés 


\title{
Le chant du monde
}

\author{
Les voix méditées-mélodiées du biographe selon Jean Grémillon
}

Philippe Roger

1 Qu'il y ait, au sein d'un art, plusieurs conceptions de cet art, cela va de soi. Que certaines soient moins répandues que d'autres, l'expérience le confirme. La manière dont Jean Grémillon conçoit le cinéma est si originale qu'elle explique pour l'essentiel l'incompréhension dont il a souffert, et qui continue à lui attacher l'image d'artiste maudit. Dès qu'on tente de comprendre un aspect de son cinéma, la nouveauté de sa vision étonne toujours. Il en est ainsi de la voix, dont il use dans ses films d'une façon telle qu'on ne peut que s'interroger sur son rapport au monde, et au monde sonore. Cette étude des fictions et documentaires de Grémillon prend en compte les écrits du cinéaste ; même s'il convient d'être prudent sur les théorisations d'un artiste, on ne peut les négliger, lorsqu'elles relèvent en ce cas d'une méditation continuée toute une vie.

On ne trouve guère de références directes au domaine vocal dans les écrits de Grémillon. Tout juste rencontre-t-on cette remarque paradoxale, d'allure provocatrice en ces débuts de cinéma parlant (1931) : « Le film parlé ou sonore, dans mon esprit, c'est la même chose, car un personnage qui parle ne m'intéresse pas davantage qu'un moteur, qu'une sirène, etc. ${ }^{1}$ » Si l'on peut y lire le refus du cinéaste de se conformer à la mode de la prééminence de la parole (il paie alors le prix fort de cette indépendance d'esprit), on doit surtout prendre au sérieux ce qui se joue dans cette affirmation, d'une conception toute personnelle du cinéma. Il y est question du sonore, non du parlant; le registre vocal le mène ainsi à celui du bruit (moteur, sirène, des bruits qui occuperont une large place dans ses films) ; pour un peu, il évoquerait aussi la musique (ce qu'il fera dans d'autres écrits). L'essentiel est le choix du terme sonore: pour Grémillon, bruit, parole et musique relèvent d'une même réalité, celle du monde sonore. Un point de vue cohérent dont il tire nombre de conséquences, à commencer par l'intime solidarité des éléments sonores. Le musicien Grémillon n'a pas attendu les balbutiements du cinéma parlant pour penser de la sorte ; n'écrivait-il pas dès 1925 : « Il y a des sonorités latentes dans le silence » ${ }^{2}$ ? Un état d'esprit dont on retrouve encore des traces en 1950: "Une musique peut être discrète tout en étant extrêmement véhémente $»^{3}$. Le lien qu'il établit entre les diverses 
données du sonore justifie son intérêt pour le mixage: "L'opération technique des mélanges est passionnante $»^{4}$.

De tels traits manifestent une pensée exceptionnellement attentive à l'univers sonore sous toutes ses formes. Une pensée qui se traduit par des positions qui peuvent surprendre, on l'a constaté pour la minoration de la parole. Afin de prendre la mesure de l'originalité d'une telle conception, il faut aussi aller voir du côté d'un autre pôle du sonore cinématographique, la musique, que Grémillon envisage également de façon paradoxale. La manière dont il marque son approbation du travail du dernier compositeur qui ait collaboré avec lui, pour son ultime long métrage, donne une idée assez exacte de sa conception : «Dutilleux a très bien compris ce qu'il fallait. Sa musique n'a pas d'expression dramatique en soi, je veux dire qu'elle ne commente ou n'explique jamais les sentiments des personnages. Elle intervient comme un élément autonome ${ }^{5}$. » De même que le cinéaste met en cause la place privilégiée de la parole ordinaire, il refuse à la musique de film son rôle habituel de pourvoyeuse facile de sentiment. Lors d'une conversation de 1950 avec son compositeur attitré, Roland-Manuel, il justifie sa position :

4 " Faut-il demander à un musicien une musique qui soit nécessairement expressive? Je réponds non, car le film est déjà de toute façon un moyen démonstratif et toute musique qui se voudrait, elle aussi, expressive, de sentiment, démonstrative, pittoresque, amène une sorte de surcharge qui fait que le spectacle qui est à la fois auditif et visuel est, à mon avis, intolérable $»^{6}$.

Roland-Manuel est du même avis : "L'émotion musicale ne s'ajoute pas à une autre émotion, l'erreur est complète $»^{7}$.

6 Voix réduite au bruit, musique conçue comme élément autonome: autant de choix inhabituels en matière de cinéma, qui prennent sens dans une forte pensée du sonore. Cette pensée seule pourra rendre compte, entre autres, de la dimension vocale de l'œuvre du cinéaste. Un texte théorique essentiel, abordant l'ensemble de ces points fut publié une vingtaine d'années après la disparition de Grémillon, dans la revue Cinéma 81. Dans cet écrit majeur, le cinéaste désigne le sonore comme le fondement de son art : " $\mathrm{La}$ cinématographie des gestes, des attitudes, des actes, ne sont que les effigies mortes auxquelles il manque le bain d'un élément où ces réalités en continuel déplacement prennent leur sens profond. Cet élément, c'est le son $»^{8}$. Le sonore serait source de vie du cinéma, un cinéma conçu comme écriture de la vie: "Il y aurait une façon directe d'attaquer le problème cinématographique; ce serait tout simplement de partir de sa base étymologique : écriture du mouvement ou écriture de la vie (cinématographe ou biographe), ce qui revient au même $»^{9}$. Parmi les grandes visions de poètes du cinéma français, à côté du cinématographe revendiqué d'un Bresson ou d'un Cocteau, une place de choix doit revenir au biographe de Grémillon. Un biographe tissé d'images et de sons solidaires : "Le sentiment de présence est au cinéma la coïncidence entre l'événement représenté et l'intention du fait sonore qui présage ou prolonge cet événement $»^{10}$. Ce fait sonore primordial, Grémillon va en définir les lois d'action, qu'il nomme vertus agissantes, prenant pour base non la voix mais le bruit, tout en les associant comme le suggère son attaque :

7 « Il y a des sons morts comme il y a des mots morts, et nous sommes amenés à nous demander ce qui constitue la vertu agissante d'un bruit. Un bruit agit-il par définition, c'est-à-dire en provoquant en nous la formation d'une image correspondant exactement à l'objet qui a produit le bruit, ou bien par allusion, c'est-à-dire en nous laissant le choix entre plusieurs images assez imprécises et indéterminées, pour que le sens qu'il prendra 
lui soit attribué par nous, ou bien agit-il par envoûtement, c'est-à-dire le bruit n'étant plus reconnaissable évoque-t-il allusivement une image en participant à la création d'un état de bien-être ou de mal-être qui prépare de ce fait le terrain à l'image que nous voulons faire surgir chez le spectateur auditeur? $»^{11}$

8 Ce spectateur-auditeur, c'est le " "regardant" du film »" Grémillon dans son article tardif sur Masson, un regardant-écoutant, faut-il préciser.

9 L'ouverture de la proposition théorique esquisse un rapprochement entre bruit et voix : $i$ $y$ a des sons morts comme il $y$ a des mots morts. $Y$ aurait-il un parallèle à établir entre les registres du bruit et de la voix ? On serait porté à le croire, tant les deux premières dimensions du travail esthétique portant sur la dimension sonore, tel que défini par le cinéaste, rappellent les distinctions classiques entre sens littéral et sens figuré d'un même vocable ; la première action sonore, par définition, relèverait d'une sorte d'univocité littérale, provoquant la formation d'une image correspondant exactement à l'objet qui a produit le bruit; quant à la deuxième action sonore, celle procédant par allusion, c'est-à-dire laissant le choix entre plusieurs images assez imprécises et indéterminées, pour que le sens qu'il prendra lui soit attribué par nous, il paraît relever de la pluralité des figures de sens possibles, de la secondarité de l'interprétation du regardant-écoutant. Là où les choses gagnent en mystère, c'est lorsqu'on accède à la troisième dimension invoquée par le cinéaste: à quoi peut correspondre l'action sonore par envoûtement? Ce qu'en dit Grémillon prend appui sur la dimension précédente, non pas définitoire (sens littéral) mais allusive (sens figuré) : le bruit n'étant plus reconnaissable évoque allusivement une image ; mais un renversement majeur s'opère : l'action sonore par envoûtement participe à la création d'un état de bien-être ou de mal-être qui prépare de ce fait le terrain à l'image que nous voulons faire surgir chez le spectateur auditeur. Ce troisième sens n'est plus le fait du regardant-écoutant récepteur, mais du concepteur "compositeur du film". Cette formule convient en la circonstance, la référence musicale s'imposant de plusieurs manières. L'idée d'envoûtement sonore traduit celle de charme musical, au sens fort; puis, la description du processus (création d'une sensation dans une visée de révélation) est assez conforme au phénomène musical ; argument décisif, Grémillon prolonge son texte quelque peu sibyllin par un développement où il n'est question que de composition sonore, par le bruit et la musique. Il distingue la moindre plasticité du bruit de celle de la musique: "Bien que les bruits puissent s'ordonner analogiquement en éléments constitutifs d'un état sonore particulier, leur matière même est beaucoup plus rebelle que celle de la musique $»^{13}$; une réserve ne devant pas faire oublier que Grémillon s'intéresse de longue date au traitement musical du bruit, notamment vocal (dès 1930 avec le cri à l'envers figurant dans la Petite Lise), ses recherches culminant dans son court métrage expérimental Astrologie ou le miroir de la vie en 1952-53, où il confie au jeune Pierre Henry le soin de composer pour quelques scènes de la musique dite concrète, autrement dit un travail musical sur du bruit enregistré. Ensuite, le cinéaste avance une définition abstraite de la composition musicale : « Dans la musique, les sons sont distribués selon une échelle et dans un ordre qui déterminent très exactement leur mode. Le compositeur de musique bouscule cet ordre et assigne aux sons un rang, un voisinage qui créera une parenté réelle à cette nouvelle famille dont les éléments sont indéfiniment amovibles $»^{14}$. Sa conclusion rapproche, au mot près, le travail du cinéaste et celui du compositeur tel qu'il vient de le définir: "L'extension des combinaisons d'images visuelles et d'images sonores est telle que le cinéaste peut revenir indéfiniment vers le connu, en faire jaillir des expressions indéfiniment neuves dans un monde qui semble demeurer éternellement vierge $»^{15}$. 
10 Travail musical que l'action sonore la plus subtile, procédant par envoûtement? Un indice capital vient le confirmer. Grémillon emploie deux termes concordants pour qualifier les trois vertus agissantes qu'il vient de définir. Evoquant " cette sorte d'alchimie ${ }^{16}$ » qu'est à ses yeux sa triple action esthétique, il parle à son propos de "la magie des sons ${ }^{17}$. " Alchimie, magie : des termes qui font écho à un autre écrit important du cinéaste, où l'on découvre ce passage explicite: "Il y a dans la musique une possibilité d'expression métaphysique que vous ne trouvez pas dans les autres arts. Cela tient aussi à cette espèce de phénomène magique qui est à la base de toute création. La magie opère mieux et plus facilement au moyen des sons qu'au moyen des images ${ }^{18}$. Ce qui précise plusieurs points. D’abord le modèle musical du biographe grémillonien : la musique est comprise comme la conscience du monde, seule expression métaphysique possible du réel ; on conçoit mieux le rejet souvent réitéré par Grémillon d'une conception sentimentale, réductrice, de l'expression musicale : il serait du devoir du cinéaste de faire accéder son public à la dimension métaphysique par l'usage raisonné de la musique de film. Puis l'insistance concomitante sur la dimension magique du processus artistique. Encore faut-il s'entendre sur le sens que Grémillon donne au terme de magie. À la question : " Ne pourrait-on pas essayer de retrouver ce processus de la magie du son au cinéma? ${ }^{19}$, le cinéaste convoque les civilisations orientales qui ont su préserver la sagesse des Anciens, dont le monde médiéval occidental témoigne aussi. «Ceux qui ont le mieux compris cela, ce sont les Chinois. Ils ont d'ailleurs non seulement un système musical parfait, mais tout un système de l'univers - une métaphysique absolument cohérente où la musique est exactement à sa place, avec des correspondances tout à fait extraordinaires $»^{20}$. Autrement dit, la vraie magie est un mode de compréhension du monde, de mise en évidence des liens unissant l'homme au cosmos. Le seul réalisme que conçoit Grémillon est magique: "C'est le chemin du réel qui me passionne. Si j'évoque l'idée "Réalisme cinématographique" je réponds : révélation d'un secret par le moyen d'une admirable mécanique qui charme autant nos yeux qu'elle ravit notre esprit et pour des raisons de vérité ${ }^{21}$. Ce terme de révélation est central. La troisième action sonore, celle procédant par envoûtement, a fonction de révélation (de ce que le cinéaste veut faire surgir chez le spectateur-auditeur). Cinéaste de la profondeur, Grémillon n'a qu'un but, qu'une raison de vérité: "Rendre sensibles les aspects secrets d'une réalité profonde » ${ }^{22}$. Sa poétique sonore, qui conjugue bruit, voix et musique, peut à ce titre se définir comme un élargissement de la perception, un agrandissement de l'écoute du monde. La voix prend en ce sens une place de choix, celle de la parole poétique, qui est nomination des êtres et des choses :

11 "Lorsque vous évoquez un créateur, qu'il soit peintre, musicien ou poète, vous faites surgir l'image d'Orphée qui appelle les choses et les éléments par leur nom afin qu'ils répondent et soient présents. C'est un don particulier que celui de nommer, car c'est l'emploi du nom propre et vrai qui, sans cérémonies et sans démons, défie les angoisses et les charmes, qui rappelle l'apparent à l'essentiel $»^{23}$.

Le don orphique du langage s'applique autant au son (musicien) qu'à l'image (peintre), maniés par le cinéaste (poète) qui rappelle l'apparent à l'essentiel. Poétique cinématographique, poétique biographique; ne pourrait-on pas rapprocher les trois niveaux d'action sonore vivante - définitoire, allusive et magique - des trois sources du son au cinéma, bruit, voix et musique : bruit définitoire, voix allusive, musique magique? $\mathrm{Du}$ «charmeur de sons ${ }^{24}$ qu'est pour lui l'homme de radio et de cinéma Maurice Cazeneuve, Jean Grémillon écrit des lignes qui semblent s'appliquer d'abord à lui-même : 
il « tentait dans chaque expérience qu'il entreprenait de reculer les limites de l'expression, d'élargir la représentation, et du même coup d'agrandir l'homme par l'image qu'il se proposait d'en montrer $»^{25}$.

13 La définition du réalisme que donne Grémillon est exemplaire de cette foi en l'art et en l'homme :

14 «Selon moi, le réalisme pourrait être la découverte du subtil que l'œil humain ou l'oreille humaine ne perçoit pas immédiatement, et qu'il faut montrer ou faire entendre en établissant des harmonies, des relations entre les objets et les êtres, en vivifiant chaque fois cette somme inépuisable de sensations visuelles et auditives qui frappe notre imagination, qui enchante notre cœur $»^{26}$.

Le biographe, cette rigoureuse écriture de la vie, entend vivifier la somme inépuisable des sensations auditives, par la triple vertu agissante du sonore. Chez Grémillon Harmonies et relations ne sont pas tout à fait synonymes. Les harmonies se réfèrent à la fonction musicale de la mise en scène cinématographique ; établir des relations entre les objets et les êtres, c'est encore agir à la façon du compositeur de musique qui réorganise les relations sonores, c'est aussi mettre l'accent sur le lien fondateur entre l'homme et le cosmos: « Découvrir ou rejoindre les secrets d'un monde qui est à la fois celui des humains et celui des choses $»^{27}$. Toujours chez le cinéaste proximité des vocabulaires musicaux et cosmologiques. Dans des notes prises en vue d'une conférence, il revient sur le sujet qui lui tient à cœur ; Grémillon distingue d'abord le réalisme du naturalisme : «A préciser : réalisme de l'expression et non réalisme du motif qui fait de l'auteur un esclave du modèle $\aleph^{28}$. Puis définit son réalisme magique :

16 « Transposition qui délivre et fait entrer l'esprit créateur dans le plan de l'univers. La vie n'est pas seulement dans l'objet... mais dans les rapports sensibles de tous les objets entre eux et aussi dans les rapports intuitifs avec celui qui les regarde. Lorsqu'un auteur de films a ce pouvoir de transposition, tout ce que son œil rencontre est transfiguré et ce que nous avons tous les jours sous les yeux devient nouveau - comme si nous le voyions pour la première fois. Son pouvoir sur la matière est assez grand pour que l'esprit y trouve son chemin et s'y montre $»^{29}$.

17 Transposer, transfigurer : ouvrir dans la matière le chemin de l'esprit! Transposition est terme musical. Le métier d'auteur de films consisterait à transposer pour transfigurer, c'està-dire faire entrer l'esprit créateur dans le plan de l'univers. C'est là le sens ultime de la tripartition des opérations sonores, qui vont permettre de suivre en leur trajectoire bruit, voix et musique dans la constellation poétique du biographe.

On ne sera pas surpris de la place majeure dévolue à la musique: celle de l'esprit, de l'organisation de la matière, de la compréhension du cosmos. Mise en lumière philosophique des rapports sensibles, la musique grémillonienne est la clef de compréhension de l'univers. En symétrie, le bruit est la figuration cosmique inverse, dans cette rêverie sonore essentielle ; le bruit est le corps opaque du monde, la matière d'allure incohérente ; le bouillonnement sonore d'une vie obscure. Bruit lunaire, ombreux, et musique solaire, lumineuse, sont les deux colonnes du temple cosmique, les deux aspects du macrocosme. Reste la voix; elle occupe une place paradoxale, à la fois périphérique et centrale; elle ne joue pas dans la même catégorie que la musique et le bruit mais participe intimement de ces deux pôles. C'est que la voix figure le microcosme, c'est-àdire l'espace de l'homme ; la voix est l'âme, est l'humain. Voix microcosmique, qui reflète le macrocosme sous ses deux aspects, du donné brut (bruit) et du pensé élaboré 
(musique); elle ne vibre pas seulement de résonances cosmiques, elle est mue en profondeur par les contradictions (bruit) et les complémentarités (musique) du macrocosme. Si pour Grémillon l'homme condense tous les mystères, polarise tous les secrets, c'est que le microcosme humain, dont la voix s'avère la plus juste des manifestations, ne relève pas d'une identité fixe mais se révèle mutable, au croisement de forces qui le meuvent et le dépassent. D'où l'idée d'un mouvement sonore général traduisant à la fois la solidarité des énergies et l'échange des qualités. Une solidarité d'abord sensible dans le microcosme. Âme tenant du corps et de l'esprit, la voix est vibration, mixité oscillatoire ; la vie de la voix tient à son origine plurielle, son impureté féconde. Quant à l'échange des qualités, il fait de la voix un bruit et une musique tout à la fois : bruire et chanter font intrinsèquement partie des manifestations vocales; les vertus de définition et d'envoûtement complètent ainsi son mode allusif. Le travail patient du cinéaste va porter sur une direction non pas tant du jeu que de la voix de ses interprètes: modulation vocale s'étendant du bruit au chant et réciproquement, originalité de sa direction d'acteur. Le courant sonore grémillonien dépasse le champ du vocal, en ce sens que le travail à l'œuvre sur la voix s'applique aux autres sources sonores. À la diversité du vocal répond l'unité du sonore, en raison du principe de solidarité ; si la voix est double, le son est un. Dans les films de Grémillon, l'échange des qualités sonores fait que tout son peut relever tour à tour ou simultanément du bruit, de la voix et de la musique, conséquence des lois de l'unité essentielle et du mouvement vital du monde. Si la voix bruit et chante, le bruit parle et chante, quand la musique bruit et parle. Le biographe entend rendre compte de cette circulation continue, d'où l'ambition extrême et la complexité irréductible de ce cinéma du vivant. Dès qu'on cherche à isoler un élément pour le définir, on rencontre les principes de solidarité et d'échange qui rendent l'entreprise délicate. Cette voix qui bruit et chante, ce bruit qui parle et chante, cette musique qui bruit et parle... Cette âme qui tient du corps et de l'esprit, ce corps qui tient de l'âme et de l'esprit, cet esprit qui tient du corps et de l'âme...

Ce que Grémillon accomplit dans la partie émergée de son œuvre, ses longs métrages de fiction, il en livre les clefs dans la partie immergée, ses courts métrages. Poétiques et didactiques, ces précieux documentaires clarifient un univers esthétique unique. On ne mentionnera que ceux qui intéressent le champ du sonore. Lorsqu'il trace le plan de son film le plus expérimental, Astrologie ou le miroir de la vie (1952-53), le cinéaste qui fait appel de façon inhabituelle à deux compositeurs pour le même court métrage, indique selon les scènes musique concrète (que composera Pierre Henry) ou musique abstraite (il désigne de la sorte la musique instrumentale composée par Marius Constant). Autant l'expression musique concrète, liée à Pierre Schaeffer, commence à circuler en ce début des années cinquante, autant celle de musique abstraite renvoie non seulement à l'opposition naissante de l'enregistré à diffuser et de l'instrumental à jouer, mais encore à la conception grémillonienne d'une musique métaphysique. Le sonore grémillonien oscillant entre le bruit concret et la musique abstraite, la voix tiendrait à la fois du physique et du métaphysique, donc au moins des deux premières dimensions de l'action sonore, par définition et par allusion; elle participerait également, de par le mouvement qui la meut, de la troisième dimension, par envoûtement, qui travaille les idées de circulation généralisée et de faculté poétique de transposition; la permutation des données du sonore relève de la transposition poétique. Par ailleurs, le lien vocal physiquemétaphysique doit être rapproché du rapport corps-esprit, tel que suggéré par une gravure alchimique de 1619 figurant dans un ouvrage de Robert Fludd, gravure citée par Grémillon dans le même documentaire : il s'agit d'une tête de profil donnant à voir deux 
yeux, l'un physique, à sa place accoutumée, l'autre mental, situé à l'intérieur du cerveau : ceil de l'imagination, dit œil de l'esprit ; allégorie proche de celle décrite au début du court métrage jumeau Alchimie, avec ses deux livres « l'un ouvert, celui des signes visibles de la Nature, l'autre qui est fermé : celui de la nature secrète des choses $»^{30}$; à ce titre, la voix évoluerait en clair-obscur entre bruit exotérique et musique ésotérique. La vision fluddienne évoque une écoute du même ordre que son double regard; le cinéma de Grémillon demande à être écouté par une oreille de l'imagination, apte à saisir entre autres les subtiles modulations vocales qui s'y jouent. Si le visible est le chemin vers l'invisible, il s'agit de même d'ouvrir dans l'audible le chemin vers l'inouï.

Ce à quoi l'on pense devant l'impressionnant dispositif vocal conçu en 1951 par le cinéaste pour un court métrage d'art qui ne verra pas le jour, l'Apocalypse de saint Sever :

«Il y a lieu, pour la continuité sonore du film, d'imaginer : 1 . Une voix de récitant pour le texte narratif; 2. Des voix amplifiées par une longue et profonde résonance pour les interventions des quatre Êtres et des Anges; 3. Un chœur parlé et un groupe d'instruments à percussion pour les allusions aux cataclysmes; 4. Des chœurs chantés, pour les interventions de l'Éternel ou des Saints, extraits des liturgies synagogale, romaine, grecque, arménienne, syriaque et copte $»^{31}$.

Il s'agit là du plus important document de travail que Grémillon nous ait laissé, sur sa pensée du sonore vocal. Un vocal jouxtant le musical : le cinéaste avait d'abord écrit la partition sonore du film, avant de biffer le mot partition pour le remplacer par celui de continuité. On trouve dans ces notes éclairantes la confirmation que le cinéaste pense le sonore dans la continuité ; du parlé au chanté en passant par le bruit, la voix s'y déploie en constantes métamorphoses. Les quatre registres vocaux imaginés par le cinéaste font passer de la parole à la musique par l'intermédiaire des bruits. On rencontre d'abord le parler simple, celui de la voix de récitant (partie qu'aurait sans doute tenue Grémillon, comme dans tous ses documentaires ; le contrat apprend qu'il se serait aussi chargé de composer la musique); voix soliste, captée de la façon la plus pure. Puis viennent les voix des protagonistes de ce drame sacré, ces acteurs de l'Apocalypse que sont les "êtres" et les anges ; ces voix se trouvent placées dans un bain qui les colorent, elles sont réverbérées dans une acoustique qui les rattache à la fois au domaine des bruits et à celui de la musique, par le jeu sur la résonance. Un exemple permettra de se faire une idée de l'impression vocale qu'aurait produite le contact des deux premiers registres; dans cet extrait, on passe de la voix du récitant à celle de l'aigle, un des "êtres" de l'Apocalypse :

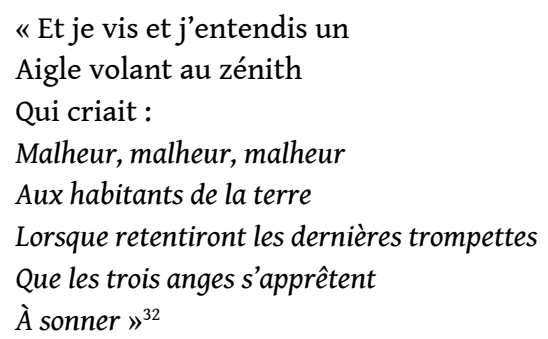

Le nombre des voix s'élargit ensuite à l'ensemble d'un chœur parlant, lui-même enrichi d'un ensemble instrumental proche du territoire des bruits, puisqu'il est composé de percussions; ce chœur accompagne des événements liés à l'idée de bruit, s'agissant de l'évocation sonore de cataclysmes. Les voix trouvent enfin leur accomplissement musical dans une série de chœurs cette fois chantés - chœurs liturgiques empruntés aux confessions les plus diverses: la musique dévoile, révèle son caractère sacré. Un tel déploiement de formes vocales peut se comprendre comme le développement projeté du 
geste sonore majeur refermant un film précédent du cinéaste : la prière des agonisants de Remorques (1941, soit une décennie avant l'Apocalypse). Le long métrage s'achevait en oratorio, unissant pas moins de trois effectifs vocaux : d'une part deux solistes à la parole psalmodique, voix de femme et d'homme, d'autre part un chœur également parlant, associé aux deux récitants, enfin un chœur vocalisant, mêlé à l'orchestre d'un bout à l'autre de la cantate. Déjà le spectre sonore était large, des récitants aux chœurs faisant usage de la parole et de la vocalise. L'Apocalypse aurait amplifié de façon décisive la création vocale grémillonienne, mais puisque ce film n'a pu se concrétiser (pour des causes semble-t-il visuelles, la pellicule couleur prévue ne donnant pas satisfaction), c'est Remorques qui reste en mémoire comme accomplissement de cette poétique de la voix.

Il est vrai que ce long métrage est l'un des plus complets du cinéaste, sur le plan de la réalisation vocale. L'oratorio final est le couronnement d'un ensemble porté par une rare cohérence. Dès la première tempête la nature se met à parler, à vocaliser plutôt : les interventions d'un chœur rythment une partition orchestrale intimement mêlée aux bruits et aux voix. La vocalité est centrale dans Remorques: quand la musique instrumentale devient vocale, le bruit se métamorphose en cri : d'abord perçue comme appel au secours, la sirène du navire se mue en cri déchirant de bête blessée à mort. Le cinéaste alterne les ambiances sonores placées sous le signe du réalisme de surface, définitoire, ou de profondeur, allusif, et les passages d'envoûtement, de magie au bord du murmure (la villa en bord de plage, la chambre d'hôtel), parfois hurlée (les deux tempêtes). La voix part du silence, malheureux car rentré (l'épouse : " Voilà des années que je me tais, alors que j'appelle au secours ») ou heureux car donné (la maitresse confiant son nom rêvé à l'oreille de l'homme), et s'aventure jusqu'au cri (le mari face à la mort de sa femme).

Remorques est à mettre en perspective avec certaines des expériences du sonore qui l'ont précédé et ont pour nom la Petite Lise et l'Étrange Monsieur Victor, soit le début de la première période parlante du cinéaste, en 1930, et le premier accomplissement sonore de sa deuxième période parlante, en 1937. La petite Lise est l'un des films les plus inventifs du début du parlant; Grémillon y fait preuve d'une rare audace dans le maniement du sonore. Déjà, il opère une distribution autant vocale que visuelle, qui confronte force et fragilité : la voix puissante et tendre du père, étranglée d'émotion, et celle de sa fille, frêle filet douloureux. Puis il intègre les voix dans un sonore continu : elles s'extraient du bruit et s'orientent vers le chant. C'est déjà le cas de la première grande scène, au bagne : de la rumeur indistincte naît un dialogue, puis une chanson reprise en chœur. Dès son premier film parlant, Grémillon donne un statut privilégié à une forme musicale considérée à tort comme mineure : la chanson ; jusqu'à son dernier long métrage, il restera fidèle à ce choix personnel. La Petite Lise s'ouvre en son générique par une mélopée exotique d'allure fataliste, chanson conduite par une voix féminine, accompagnée par les vocalises d'un chœur masculin ; le bal antillais final voit le retour d'un chant réduit à son rythme. Et les bagnards entonnent un air 1900, Ferme tes jolis yeux, que citera fugitivement le cinéaste dans Lumière d'été, film dont le sommet dramatique correspond aux vocalises inquiétantes d'une soliste anonyme, lors de la farandole fatale reprenant une mélodie de Rossini. Le chant populaire, la chanson, tend souvent chez Grémillon à se confondre avec la mélodie savante. Un des plus beaux exemples, avant la cantate honeggerienne de Remorques, est donné au retour de Bastien à Toulon dans l'Étrange Monsieur Victor: Roland-Manuel associe au paysage visuel un paysage sonore, d'une voix féminine soliste chantant une 
mélodie dans un style populaire archaïsant, aux accents quasi ravéliens, et d'un chœur vocalisant. Cette musique vocale dit l'indicible, de l'âme du lieu.

A côté de ces grands moments, Grémillon parsème à même fin ses films de voix anonymes qui peuplent les espaces publics, cours de Paris, rues d'Orange et de Toulon: cris de marchands ambulants, ils émanent des lieux comme s'ils en exprimaient l'âme. Et c'est le cas. Peu intelligibles, ils n'ont pas vraiment de fonction utilitaire : toujours au bord de la vocalise - figure musicale obsédante de cette poétique sonore -, on n'en perçoit guère le sens. Le plus travaillé est celui hantant la cour de l'usurier dans la Petite Lise. Grémillon fait entendre à l'envers le chant de cette voix, perçue étrangère parce qu'étrange; le procédé n'est pas gratuit, qui suggère la magie dangereuse de l'endroit, ce lieu du crime imminent. Quelle que soit la situation, le cinéaste confie à la voix anonyme, parlée ou chantée, le soin de rendre sensible l'esprit du temps et du lieu.

L'exemple le plus abouti d'une chanson-mélodie traduisant l'âme d'un paysage, d'un pays même, on le rencontre dans le Six juin à l'aube ; à l'été 1946, Grémillon compose la musique de son documentaire le plus ambitieux. Le cinéaste part d'une chanson française du treizième siècle (on sait la grande culture médiévale de Grémillon), Ce fut en mai du trouvère Moniot d'Arras, chanson qu'il fait d'abord entendre selon sa disposition favorite (voix féminine avec chœur de vocalises), puis transpose à l'orchestre seul dans un rythme très contemporain, enjambant près de sept siècles. Aux jeux temporels s'adjoignent souvent des jeux de sens. Le choix du cinéaste de privilégier musicalement la forme vocale de la mélodie (au sens de lied), le conduit à des jeux très libres entre le texte et la musique des chansons qu'il cite dans ses films. On en trouve des exemples remarquables dans le Ciel est à vous, aussi dans l'Amour d'une femme une décennie plus tard. Deux chansons structurent le Ciel est à vous : Sur le pont du Nord (ou de Nantes) et Le temps des lilas et des roses. Chansons à la musique lumineuse, allègre, mais au texte sombre, désespéré (dans le premier, deux enfants se noient pour avoir désobéi à leur mère; le second est une déploration sur l'amour évanoui ${ }^{33}$ ). Les menaces tapies dans les paroles sont masquées par la musique. Le recours fréquent aux vocalises leur confère une fonction de masquage, d'autant que le cinéaste ne propose que les paroles les moins explicites. Il en va de même pour l'Amour d'une femme; les hommes du chantier ouvrent et ferment presque le film par une chanson à boire du dix-huitième siècle, due au comte de Bonneval : Nous n'avons qu'un temps à vivre. En changeant un terme (amis devient compagnons) Grémillon en indique discrètement ce qui lui paraît être le sens maçonnique, tout en retranchant l'innocente charge contre l'alchimie que contenait la chanson ${ }^{34}$.

Le souffle vocal comprend pour Grémillon à la fois les tremblements de terre du bruit et les courants des eaux de la musique. La voix tient d'abord du bruit par sa matière, sa granulosité ; le cinéaste joue en expert des textures vocales, des plus lourdes, mêlées, aux plus légères, pures, pour dessiner la géométrie des rapports entre ses personnages. Que la rencontre avec Jean Gabin (Gueule d'amour, 1937) ait été décisive pour Grémillon se conçoit: le cinéaste peut prolonger son rêve d'une direction vocale des comédiens. Il travaille la voix de Gabin dans le sens d'une pureté sensible, musicale du piano au forte; à cette pureté absolue, le cinéaste oppose sciemment l'artificialité de la voix de la femme (Mireille Balin, toute de fausseté jouée). Dans le film suivant, la duplicité de Raimu passera par son accent méridional surjoué, que Grémillon oppose à la pureté, sobre et juste, de l'épouse de Raimu: Madeleine Renaud. Remorques (1939-41) reprend les deux voix pures de ces films, plus fragile cette fois pour Madeleine Renaud, plus virile pour Gabin ; l'écart des voix dit celle du couple. Lumière d'été (1943) oppose deux voix franches 
et pures ${ }^{35}$ (Michèle et Julien) à deux voix calculées (dans la préciosité constante pour le fin Paul Bernard, dans une tension instable pour une Madeleine Renaud que Grémillon fait changer de registre) ; quant à la voix de Pierre Brasseur, le cinéaste l'isole dans une théâtralité qui scelle sa rupture avec Michèle. La fin du film est forte, qui signe la défaite de Madeleine Renaud en recouvrant son cri par celui du sifflement de la locomotive de chantier : la voix retourne ainsi au bruit. Après les oniriques Remorques et Lumière d'été, Le ciel est à vous (1944) fait le pari de voix pures mais incarnées: Charles Vanel et une nouvelle fois Madeleine Renaud sont chargés de relever le défi ; le cinéaste forge ainsi des voix qui gagnent encore en humanité. Avec Pattes blanches (1949), le défi est autre : les deux voix pures du film se trouvent opposées dans leur rapport à la lumière : voix pure mais sombre de Michel Bouquet, voix pure et claire d'Arlette Thomas (c'est le couple du Printemps de la liberté non tourné mais radiodiffusé). La seule chose à sauver de l'exsangue Étrange Madame X (1950) est justement la voix pure d'Arlette Thomas. L'inégal Amour d'une femme (1954) souffre pour sa part d'une coproduction franco-italienne qui mène au doublage conventionnel de l'acteur principal, Massimo Girotti ${ }^{36}$; le doublage de Paolo Stoppa, dans la courte mais essentielle scène de l'oraison funèbre, se révèle un pur moment de grâce, le cinéaste prêtant sa voix au curé d'Ouessant. Voix d'une simplicité conquise, sobre et poétique, à la psalmodie au bord du chant; voix doucement chantante, à peine ombrée de tristesse, dont les appuis, les relances et les élans viennent rythmer une scène dont le drame latent est marqué par un souffle inquiétant qui vient s'opposer à celui de l'homme : celui de la tempête mauvaise qui se lève; voix chaude du microcosme contre vent froid $\mathrm{du}$ macrocosme. Le film offre par ailleurs des variations vocales intéressantes; le cinéaste manifeste à nouveau son goût pour les contrastes vocaux : la voix haut perchée de Gaby Morlay tranche sur celle plus neutre de Micheline Presle. Poignante aussi, l'expression vocale d'une vie en danger : afin de rendre sensible la lutte de la vie contre la mort, Grémillon fait entendre en continu le faible halètement agonique de la petite fille fiévreuse ; alors que son souffle traduit le combat incertain en cours, le silence qui suit manifeste paradoxalement la victoire de la vie: la malade s'est mise à dormir paisiblement, sans bruit.

L'oraison funèbre prononcée par le curé d'Ouessant est la seule occurrence repérable de la voix du cinéaste dans son œuvre de fiction; elle n'en est pas moins significative pour comprendre les enjeux de sa poétique vocale. Elle constitue la part émergée d'un continent sonore oublié, celui formé par ses documentaires s'échelonnant de 1944 à 1959 : Jean Grémillon s'est attribué le rôle du récitant pour l'ensemble de son œuvre documentaire d'après-guerre. Du Six juin à l'aube à André Masson et les quatre Éléments, cet ensemble compte neuf films essentiels (auquel on pourrait joindre un dixième, mineur, un court métrage politique auquel le cinéaste prêta sa voix, le Choix le plus simple $e^{37}$ ). Que disent ces films d'art, sur l'invention d'une voix? Car il s'agit bien de cela: le cinéaste invente une voix qui accomplit son grand rêve poétique. Si l'on prête attention à cette voix unique, distincte de celles que pratiquent ses confrères en documentaire à la même époque, on peut dire ceci : la voix de récitant selon Grémillon rend compte d'une nature plurielle de la voix de cinéma, une nature complexe toutefois réductible à une série de dualités, de différences conçues complémentaires. C'est d'abord le couple parlé/chanté. Quand Grémillon interprète la voix de récitant, il travaille de façon musicale à la française, pratiquant l'intrication du parlé et du chanté; entre autres choses, le cinéma de Grémillon est une réflexion personnelle sur l'art vocal propre à sa langue, maternelle. Tout en s'en inspirant, le sprechgesang ${ }^{38}$ germanique a occulté pour les historiens de la musique la spécificité française de ce délicat procédé expressif ; spécificité qui sut s'épanouir au 
tournant des dix-neuvième et vingtième siècles, autant dans la chanson dite réaliste que dans la mélodie française la plus savante. Écouter la voix de Grémillon, c'est retrouver l'esprit d'une diction de la nuance, de la variation subtile, infime mais sensible, de la retenue classique - catégorie esthétique majeure pour le cinéaste, qui cite régulièrement Rameau et Gide à son sujet. C'est là l'esprit des mélodies de Fauré, Duparc, Debussy, Ravel, Hahn, Poulenc: le plus d'expression dans l'écart le plus réduit. Le maximum dans le minimum ; le macrocosme dans le microcosme. Ce qui revient pour le cinéaste à instaurer un lien intime entre la forme du récitatif visible (du récitant de ses films) et celle de l'aria invisible (du chant pensé comme l'implicite de la voix). Un tel travail intervient dès l'écriture de la voix de récitant : l'exigence d'une langue rigoureuse est poussée loin par le cinéaste, exigence qui s'accomplit dans une prose poétique. Au-delà de cette expression achevée, où la simplicité aboutie s'allie à la densité cristallisée, il faut aller voir du côté de sa conception: Grémillon ne sépare jamais la réflexion de l'émotion; il se revendique d'une école de pensée médiévale, d'avant le cartésianisme. Le pensé se nourrit chez lui du ressenti : sa voix demeure toujours sensible. S'il fallait nommer ce qui fait le prix de sa vision vocale, il faudrait parler de pensée lyrique et, en dernière analyse, du couple notionnel méditation/mélodie. Chez Grémillon, non seulement le monde parle, mais il chante. Jusque dans le langage de l'homme. L'expression vocale traduit l'alliance rare du médité et du "mélodié" (en ancien français, mélodier veut dire chanter ${ }^{39}$ ). La voix médite et mélodie de conserve dans ces films-essais qui approchent de la perfection. Ce méditémélodié n'est jamais aussi sensible que dans la voix de récitant tenue par le cinéaste. La voix que Grémillon dirigea le mieux fut la sienne.

Le vocal est toujours au centre de sa poétique. Il est d'ailleurs arrivé à Grémillon de construire un film autour d'une voix, de chanteur autant que de comédien. C'est le cas de Pour un sou d'amour (1932) qui tient par la voix d'André Baugé, authentique baryton léger ; tessiture intéressante, car elle tient à la fois de la matière, grave, et de l'esprit, aigu. La voix de Baugé se dégage de la gangue du bruit pour s'élancer vers le ciel vocal : voix spirituelle, pour tout dire, même s'il faut attendre le film suivant pour aborder frontalement le registre du sacré : la Dolorosa (1934) relève d'un genre rare, le musical religieux. Le sommet de ce film est le duo chanté de Rafael (Agustin Godoy) et Dolores, duo mystique dont Grémillon soigne la mise en scène en trois plans révélateurs: le premier, correspondant au tutti de l'introduction orchestrale, présente l'église du monastère et son clocher; le deuxième, avec un solo d'instrument à vent, montre le reflet dans l'eau de la même église, donc inversée; le troisième, qui débute par une brève ouverture au noir, est le reflet redressé du plan précédent, l'église retrouvant son aspect premier mais en tant que reflet, le tout combiné à une surimpression de Dolores tandis que s'élève la voix de Rafael. Ces trois phases visuelles (image directe, inversion du reflet puis reflet redressé) naissent du déploiement vocal, tout en précisant la nature magique du chant, et correspondent qui plus est aux trois actions sonores définies par le cinéaste : définition, allusion, envoûtement. On gardera en mémoire le fait que le cinéaste réserve l'intervention vocale au plan magique, le troisième. Le deuxième film espagnol de Grémillon, Centinela alerta (1936), tient comme Pour un sou d'amour par la présence d'un acteur-chanteur, Ángel Sampedro Montero, dit Angelillo; c'est même le seul élément crédible d'un film bâclé qu'acheva Buñuel ${ }^{40}$. Comme celle de Baugé, la voix d'Angelillo passe d'un registre viril à des envolées quasi féminines: c'est cette hétérogénéité fondatrice, ce partage sinon ce trouble d'identité, qui aimante Grémillon lorsqu'il filme des voix. Mieux qu'aucun autre aspect, la voix rend compte du mystère humain, ce fragile 
microcosme mu par l'attraction d'un cosmos tour à tour chaotique (bruit) ou ordonné (musique) selon le point de vue qu'on porte sur lui.

L'un des points les plus curieux de cette poétique vocale demeure celui des vocalises. Leur présence récurrente au long de l'œuvre interroge. Qu'on pense aux fictions, par exemple aux vocalises de la Petite Lise, Gueule d'amour, l'Etrange Monsieur Victor, Remorques, Le ciel est $\grave{a}$ vous, Pattes blanches et l'Amour d'une femme (inventaire non exhaustif) ou bien aux documentaires, à commencer par les vocalises saisissantes du Six juin à l'aube, presque fantastiques, et transcendantes. La réponse serait sans doute à chercher dans ce qu'on a tenté de définir comme le médité-mélodié grémillonien, dont on pourrait faire remonter l'origine au chant grégorien et ses répons en fredons. Par quelque bout qu'on prenne la poétique du cinéaste, on est conduit au monde médiéval.

\section{BIBLIOGRAPHIE}

AGEL Henri, Le Tragique dans l'œuvre de Jean Grémillon, Thèse de doctorat, Paris, Sorbonne, 1971 [inédit].

BAZIN André, « Quand le cinéma fait attention à la peinture », Le Parisien libéré n²257, 17 décembre 1951.

BERTHOMÉ Jean-Pierre, «L'alchimie de l'art: Grémillon et le court métrage » in Le court métrage français de 1945 à 1968, De l'âge d'or aux contrebandiers, éditions des Presses Universitaires de Rennes, 2005.

DELORME Stéphane, « Passion Grémillon », Cahiers du cinéma n693, octobre 2013.

GRÉMILLON Jean, Le cinéma ? Plus qu'un art !... Écrits et propos (1925-1959), textes rassemblés par Pierre Lherminier, L'Harmattan, 2010.

GRÉMILLON Jean, « [Réflexions au pied d'un arbre] », L’Arc n², Aix-en-Provence, printemps 1958.

GRÉMILLON Jean, « Susciter le réel et le rendre présent », Cinéma 81 n²75, novembre 1981.

GUIGUET Jean-Claude, « Jean Grémillon cinéaste de l'essentialité », Cinéma 81 n²76, décembre 1981.

KAST Pierre, «Exercice d'un tragique quotidien : notes sur l'œuvre de Jean Grémillon », La Revue du cinéma n 16 , août 1948.

LEENHARDT Roger, «Pattes blanches », allocution à Objectif 49 (février 1949) in Chroniques de cinéma , éditions de l'étoile, 1986.

NUTTENS Jean-Dominique, « Lumière d'été, le bal des ardents », Positif n639, mai 2014.

ROGER Philippe, Le Mystère de l'Euvre : Remorques de Jean Grémillon, Lyon, éditions du Cosmogone, 1998.

ROGER Philippe, «Stylum vertere : le style décanté de Jean Grémillon, une esthétique de l'effacement », Études cinématographiques n65, 2000. 
ROGER Philippe, « Liens prosaïques et liaisons poétiques : Jean Grémillon, Remorques », Vertigo n 21, 2001.

ROGER Philippe, « De profundis clamavi, la voix liturgique de Jean Grémillon », Positif n494, avril 2002.

ROGER Philippe, « Un cinéaste qui agrandit le monde : Jean Grémillon à l'œuvre sur ses Quatre éléments » in Le court métrage français de 1945 à 1968, De l'âge d'or aux contrebandiers, éditions des Presses Universitaires de Rennes, 2005.

ROGER Philippe, «Jean Grémillon » in Dictionnaire de la pensée du cinéma [A. de Baecque et Ph. Chevallier, dir.], Paris, Presses Universitaires de France, 2012.

ROGER Philippe, «Jean Grémillon ou la poésie de l'absolu », Cahiers du cinéma n693, octobre 2013. ROGER Philippe, «L'image selon Jean Grémillon, une approche en neuf films de la poétique du cinéaste », Hippocampe $\mathrm{n}^{\circ} 10$, printemps 2014.

ROGER Philippe, «Les racines du ciel ; présence du sacré dans l'œuvre de Jean Grémillon », HMC ( Histoire, Monde et Cultures religieuses), éditions Karthala, 2015.

ROGER Philippe, « L'œil camérique, l'ésotérisme "biographique” de Jean Grémillon », Politica Hermetica, 2015.

ROGER Philippe, «Lumière de Grémillon », in Jean Grémillon et les quatre éléments, Actes du colloque de Cerisy-la-Salle d'août 2013 [à paraître].

SADOUL Georges, « Jean Grémillon ou la rigueur », Les Lettres françaises n801, 3 décembre 1959.

SELLIER Geneviève, Jean Grémillon, le cinéma est à vous, Paris, Méridiens Klincksieck, 1989.

VIALLE Gabriel, « La nostalgie d'un très lointain pays natal », La Revue du cinéma n²43 novembre 1970. VECCHIALI Paul, « Les deux mondes », Cinéma 81 n²76, décembre 1981.

\section{NOTES}

1. Jean Grémillon, « Muet et parlant », in Ciné-Journal du 6 mars 1931, repris in Le cinéma? Plus qu'un art !, Paris, l'Harmattan, 2010, p. 27.

2. Jean Grémillon, «Propositions », in Comœdia du 27 novembre 1925, repris in Le cinéma? Plus qu'un art !, Paris, l'Harmattan, 2010, p. 26.

3. Jean Grémillon, «Sur la musique de film », entretien public avec Roland-Manuel, 18 mars 1950, repris in Le cinéma? Plus qu'un art !, Paris, l'Harmattan, 2010, p. 84.

4. Jean Grémillon, Ibid., p. 87.

5. Jean Grémillon, "Sur l'Amour d'une femme ", in $\mathrm{n}^{\circ} 10$ de Positif, printemps 1954, repris in Le cinéma? Plus qu'un art !, Paris, l'Harmattan, 2010, p. 116.

6. Jean Grémillon, «Sur la musique de film », op. cit., p. 81.

7. Propos de Roland-Manuel in « Sur la musique de film », Ibid., p. 82.

8. Jean Grémillon, Susciter le réel et le rendre présent, s.d., in Le cinéma? Plus qu'un art!, Paris, l'Harmattan, 2010, p. 100.

9. Jean Grémillon, Ibid., p. 99.

10. Jean Grémillon, Ibid., p. 101.

11. Jean Grémillon, Ibid., pp. 101-102.

12. Jean Grémillon, « Ma rencontre avec André Masson », 10 mars 1958, repris in Le cinéma? Plus qu'un art !, Paris, l'Harmattan, 2010, p. 126.

13. Jean Grémillon, Susciter le réel et le rendre présent, op. cit., p. 102. 
14. Jean Grémillon, Ibid.

15. Jean Grémillon, Ibid.

16. Jean Grémillon, Ibid.

17. Jean Grémillon, Ibid., p. 101.

18. Jean Grémillon, "Grandeurs et servitudes du cinéma », entretien de début 1944 repris in Le cinéma ? Plus qu'un art !, Paris, l'Harmattan, 2010, p. 33.

19. Jean Grémillon, Ibid.

20. Jean Grémillon, Ibid., pp. 33-34.

21. Jean Grémillon, « Le réalisme au cinéma », texte du 17 juin 1952, diffusion RTF le 25 juillet 1952, repris in Le cinéma? Plus qu'un art !, Paris, l'Harmattan, 2010, p. 92.

22. Jean Grémillon, « Ma rencontre avec André Masson », op. cit., p. 125.

23. Jean Grémillon, Ibid., p. 124.

24. Jean Grémillon, «Maurice Cazeneuve, du son à l'image ", 1949, repris in Le cinéma ? Plus qu'un art !, Paris, l'Harmattan, 2010, p. 227.

25. Jean Grémillon, Ibid.

26. Jean Grémillon, «Le réalisme au cinéma », op. cit., p. 91.

27. Jean Grémillon, Susciter le réel et le rendre présent, op. cit., p. 100.

28. Jean Grémillon, "Réalisme et poésie dans le cinéma français ", notes préparatoires aux conférences de 1957, reprises in Le cinéma ? Plus qu'un art !, Paris, l'Harmattan, 2010, p. 234.

29. Jean Grémillon, Ibid.

30. Voix du récitant d'Alchimie (1952).

31. Jean Grémillon, tapuscrit de l'Apocalypse de saint Sever, Fonds Grémillon, Bibliothèque nationale, JG 44 bis 004.

32. Jean Grémillon, Ibid.

33. La référence n'est pas tant le poème d'Aragon (1940) que celui de Maurice Bouchor (1875), extrait des Poèmes de l'amour et de la mer, que mettront en musique plusieurs compositeurs français. Le poème de Bouchor d'achève ainsi : «Le temps des lilas et le temps des roses/Avec notre amour est mort à jamais ».

34. Voici le passage omis : Qu'un avide chimiste exalte / Sa fortune en cherchant de l'or / J'ai ma pierre philosophale / Le cœur d'une femme est mon trésor.

35. L'idée de "pureté vocale" veut ici traduire l'une des constantes esthétiques du cinéaste (prônant le classicisme), d'accomplissement de l'expression dans une simplicité conquise. Simplicité qui va de pair avec une luminosité intérieure. Son chef opérateur Louis Page rapporte les propos de Grémillon, pour l'idéal du cadre de l'image : Souvenez-vous de la bille d'agate. Page commente ainsi cette formule sibylline: «Je pense que pour lui, la bille d'agate c'était l'expression parfaite de la forme » (Propos de Louis Page recueillis par Armand Panigel en 1969 pour la Télévision française).

36. Le doublage de Massimo Girotti est dû à Claude Bertrand, comédien spécialisé dans le doublage, ce qui accuse l'aspect conventionnel de la voix.

37. Signé Henri Aisner, ce film de propagande fut réalisé en 1951 à l'instigation du Parti Communiste français. Grémillon intervint non seulement en tant que récitant, mais il participa très probablement à la rédaction du commentaire, et prêta plusieurs plans de son Six juin à l'aube. 38. Le parlé-chanté que nombre de compositeurs germaniques appliqueront dans leurs œuvres.

39. Il faudrait remettre en usage ce verbe mélodier, qui s'applique si bien à l'entreprise vocale du cinéaste : un idéal proche de la mélodie française, dans son parler-chanter à la fois populaire et savant.

40. Quand la voix principale n'est pas à la hauteur, le film musical s'en ressent : le maigre filet de voix du fade Henri Garat qui pousse la chansonnette dans Valse royale (1935) fait perdre toute crédibilité à ce divertissement appliqué. 


\section{RÉSUMÉS}

Jean Grémillon emploie la voix dans ses films de manière très différente de ses collègues. C'est l'ensemble du sonore - bruits, voix et musique - qu'il imagine de façon originale. Son traitement s'inscrit dans une vision du monde : Grémillon conçoit le cinématographe comme un biographe, une écriture de la vie. Cette vie est comprise sur deux plans: celui de l'homme et celui de l'univers. La voix selon Grémillon serait le reflet dans l'homme d'un macrocosme perçu comme énigme (le bruit) ou révélation (la musique). Principe pluriel, le vocal est pour le cinéaste le domaine d'un travail rigoureux, pétri de grand classicisme, entre le pensé et le ressenti, le parlé et le chanté, le médité et le mélodié. L'œuvre multiple de Jean Grémillon, fictions et documentaires, prouve la vitalité de cette conception de la voix au cinéma, qui sut s'incarner au mieux dans celle du cinéaste, récitant pour nombre de ses courts métrages d'art.

Jean Grémillon uses the voice in his films very differently compared to his colleagues. He imagines the whole sound - sound, voice and music - in an original way. His processing is part of a vision of the world: Grémillon's approach of the cinema is like a biographer's one, that is to say writing about life. This life is understood on two levels: man and universe. According to Grémillon voice would reflect in a man macrocosm perceived as an enigma (the noise) or a revelation (the music). Plural principle, to the director voice is the field for a rigorous work, full of great classicism, between thought and feeling, the spoken and sung, the melody and meditated. Jean Grémillon's manifold work, fiction and documentary work, proves the vitality of the voice conception in cinema, which knew how to embody at best in the filmmaker, reciting for many of his short art films.

\section{INDEX}

Mots-clés : Voix, Bruit, Musique, Cinéma du vivant, Vertus agissantes, Grémillon, Biographe

\section{AUTEUR}

\section{PHILIPPE ROGER}

Maître de conférences en études cinématographiques à l'Université Lumière Lyon 2, Philippe Roger est l'auteur d'ouvrages consacrés à des films de Jean Grémillon, Max Ophuls, Edmond T. Gréville, Gérard Blain et Jean-Claude Guiguet. Il a également signé un ouvrage d'ensemble sur la façon dont la ville de Lyon a été filmée. Critique à Jeune cinéma, où il tient une chronique régulière, Philippe Roger réalise des films documentaires et a coordonné avec Michel Serceau le numéro de CinémAction consacré aux Archives du cinéma et de la télévision. 